\title{
REAMPLIFICATION OF DIFFERENTIAL DISPLAY PRODUCTS: MORE IS NOT BETTER
}

\author{
Joseph B. Domachowske ${ }^{1}$ and Harry L. Malech \\ Laboratory of Host Defenses, National Institute of Allergy and Infectious Diseases, National Institutes of Health \\ Building 10 Room 11N112, 9000 Rockville Pike, Bethesda, MD 20892
}

\section{TABLE OF CONTENTS}

1. Abstract

2. Introduction

3. Materials and Methods

4. Results and Discussion

5. Acknowledgment

6. References

\section{ABSTRACT}

Differential display polymerase chain reaction is technically challenging at a number of steps, including reliable reamplification of differentially expressed sequences after they are isolated from an acrylamide gel. As the only source of the sequence found to be of interest is the gel from which it was identified as being differentially expressed, failed attempts at reamplification can be particularly frustrating. In our laboratory, attempts to reamplify DNA sequences cut from a denaturing 6\% acrylamide gel consistently failed when greater than 5 microliters of eluted product was used as template in a subsequent PCR reaction. If less template was used in subsequent PCR reactions, reamplification was consistently successful. This observation emphasized the importance of using limited amounts of template when reamplifying sequences that are differentially displayed on denaturing acrylamide gels.

\section{INTRODUCTION}

Differential display was developed as a method to identify differences in gene expression among eukaryotic cells. (1) Different primer combinations are used in a reverse transcriptase-polymerase chain reaction (RT-PCR) to generate cDNAs from mRNAs expressed in a given cell. By comparing the cDNAs derived from multiple cell types, or from a single cell type under different conditions, it is possible to detect differences in transcription products derived from the different conditions. These differentially expressed products are identified on an acrylamide gel, excised, eluted, re-amplified,and eventually sequenced (2, 3,4 ). This process can be technically challenging at a number of steps, and loss of a differentially expressed band because of failed attempts at reamplification can be particularly frustrating.

Recived 6/10/97 Accepted 7/1/97

${ }^{1}$ To whom correspondence should be addressed at: Laboratory of Host Defenses, National Institute of Allergy and Infectious Diseases, National Institutes of Health, Building 10 Room 11N112, 9000 Rockville Pike, Bethesda, MD 20892 Tel:(315) 445-2663 Fax: (301) 402-4369 E-mail:jdomach@helix.nih.gov
In our laboratory, attempts to reamplify DNA sequences cut from a denaturing 6\% acrylamide gel consistently failed when greater than 5 microliters of eluted product was used as template in a subsequent PCR reaction.

\section{MATERIALS AND METHODS}

Total RNA was extracted from 5 million K562 cells grown for three days in the absence and presence of nitric oxide as previously described. (5)

1. Duplicate cDNA synthesis reactions (Boehringer Mannheim 1st strand cDNA synthesis kit were performed using downstream primer 9 (T11GG) as the anchoring primer (Display Systems Differential Display kit, PGC Scientific, Gaithersburg, MD), according to the manufacturers instructions.

2. PCR was performed using the same anchoring primer and one of 4 different random upstream primers $(10$, 11, 12, and 13) supplied in the Display Systems kit. PCR was performed in a Perkin Elmer 9600 thermocycler using the following conditions: $94^{\circ} \mathrm{C}$ for 3 minutes, then 40 cycles of $94^{\circ} \mathrm{C}$ for 20 seconds, $40^{\circ} \mathrm{C}$ for 20 seconds, $72^{\circ} \mathrm{C}$ for 30 seconds followed by a final elongation at $72^{\circ} \mathrm{C}$ for 5 minutes. Trace amounts of ${ }^{35} \mathrm{~S}$-dATP was included in the PCR master mix per manufacturers protocols.

3. Amplified fragments from the two cDNA fractions were separated on a $6 \%$ denaturing acrylamide gel, transferred and dried onto Whatmann $3 \mathrm{M}$ paper and exposed to film for 10 days.

4. Differentially expressed bands were cut from a $6 \%$ denaturing acrylamide gel and eluted into 100 microliters of water by boiling for 15 minutes.

5. The eluted DNA samples were then used as templates for PCR amplification. 2.5 to 10 microliters of the eluted product was used in a 50 microliters PCR reaction containing 5 microliters $10 \mathrm{x}$ PCR buffer with $15 \mathrm{mM} \mathrm{MgCl}_{2}$ (Boehringer Mannheim, Indiannapolis, IN) 5 microliters 500 micromolar dNTPs (Boehringer Mannheim), 2 microliters 25 micromolar downstream primer 9 (Display Systems), 25 microliters 2 microM 

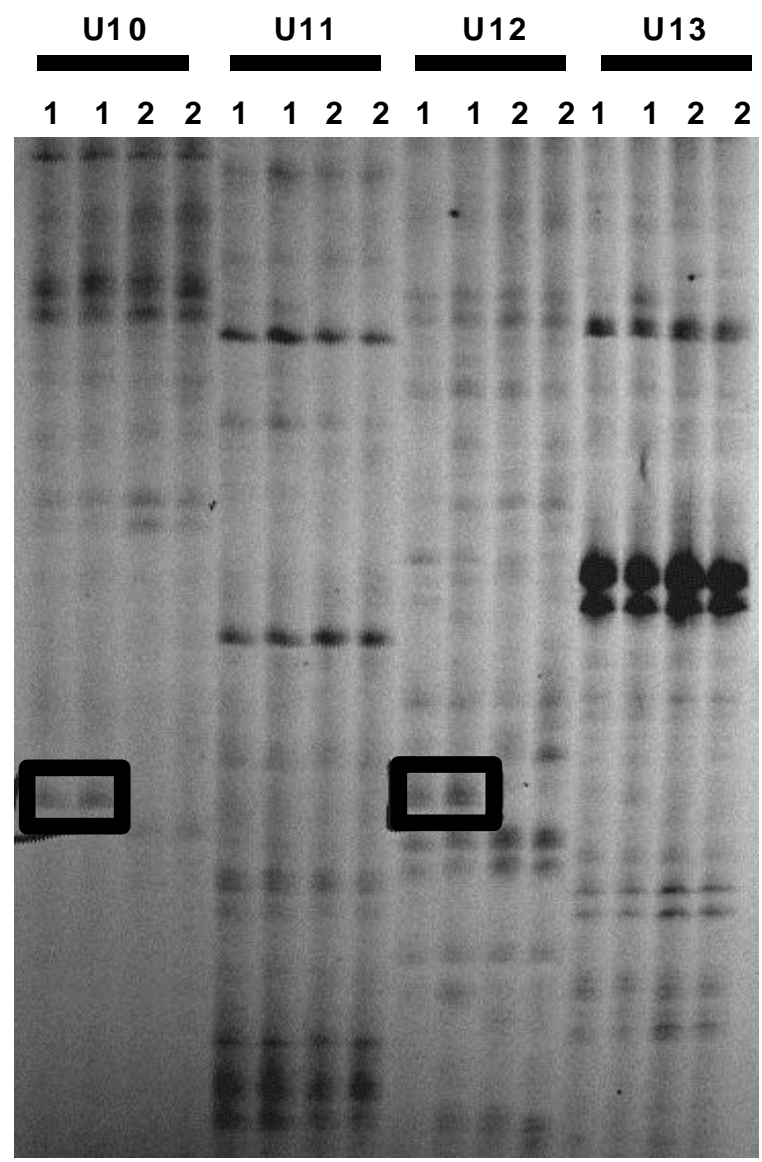

Figure 1. Autoradiograph of a differential display gel. Radiolabeled differential display PCR products from downstream primer T11GG and 4 random upstream primers, U10-13, using cDNA derived from cells grown in the absence (condition 1) or presence (condition 2) of chronic nitric oxide exposure. Boxes highlight two PCR products found to be differentially expressed.

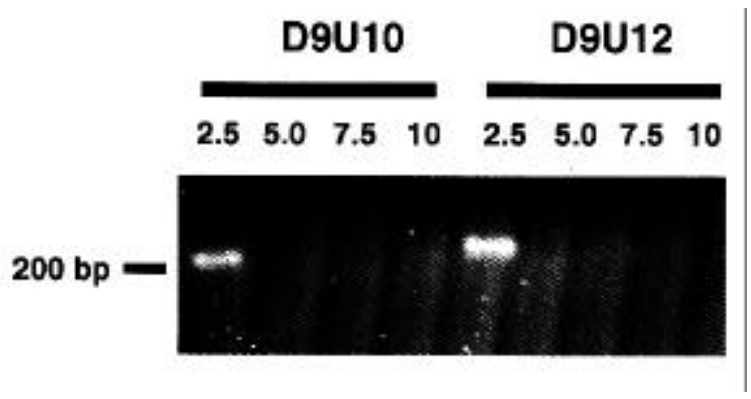

Figure 2. Reamplification of the differentially expressed DNA fragments. DNA fragments identified in Figure 1 were cut from the gel, and eluted in 100 microliters water by boiling for 15 minutes. PCR reactions were performed as described using 2.5 to 10 microliters volumes of these eluents; the products were separated on a $2 \%$ agarose gel and stained with ethidium bromide. D9 indicates the anchoring primer, and U10 and U12 are the two upstream primers, respectively. upstream primer 10 or 12 (Display Systems), and 0.5 microliters Taq polymerase (Boehringer Mannheim). Cycling conditions were identical to those used in step 2.

6. PCR products were electrophoresed in a $2 \%$ agarose gel and stained with ethidium bromide.

\section{RESULTS AND DISCUSSION}

The autoradiograph in Figure 1 shows an example of differential display results obtained using Display Systems Differential Display kit (PGC Scientific, Minneapolis, MN). In this experiment, total RNA was obtained from K562 human erythroleukemia cells (ATCC, Rockville, MD) grown in the absence (condition 1) or presence (condition 2) of chronic nitric oxide exposure (5). Duplicate cDNA synthesis reactions were performed using downstream primer 9 (T11GG) as the anchoring primer and PCR performed as described. Amplified fragments from the two cDNA fractions were separated on a $6 \%$ denaturing acrylamide gel, transferred and dried onto Whatmann $3 \mathrm{M}$ paper and exposed to film for 10 days. Two differentially expressed sequences were identified, D9U10 and D9U12. Both appear to be expressed under condition 1, but not condition 2 as seen highlighted in Figure 1. The two highlighted differentially expressed bands were cut from the gel and eluted into 100 microliters of water by boiling for 15 minutes. The eluted DNA samples were then used as templates for PCR amplification. Initial attempts to reamplify from these eluted samples, using 12.5 microliters as template in a new 50 microliters PCR reaction consistently failed. For the trial shown in Figure 2, 2.5 to 10 microliters of the eluted product was used in a 50 microliters PCR reaction. As seen in Figure 2, reamplification was accomplished for both products only when 2.5 microliters of template was used, while larger amounts of eluted sample consistently failed to re-amplify. Limiting the volume of eluted DNA sample in subsequent PCR reactions was the only modification that reliably and consistently led to reamplification in 52 separate instances (unpublished results). The biochemical basis for our observation is unknown, but the presence of inhibitors of Taq polymerase in the eluted DNA preparation, such as urea (1) cannot be excluded. This method of reamplification avoids the ethanol precipitation step of the eluted DNA fragment used by some investigators $(2,4)$.

Differential expression of the two sequences reported here was confirmed by Northern analysis. An mRNA Northern blot probed with the radiolabelled D9U10 sequence is shown in Figure 3. Only cells grown in the presence of nitric oxide trans-gene expression express a 4 kilobase mRNA identified using this probe. Beta actin mRNA signal is shown as a control.

Differential display is a challenging technique to master. The reamplification of DNA sequences eluted from denaturing acrylamide gels must be consistent and reliable. When attempting to reamplify these valuable sequences, it should be kept in mind that including more of the eluted DNA sample in the reamplification PCR mixture is not necessarily better. 


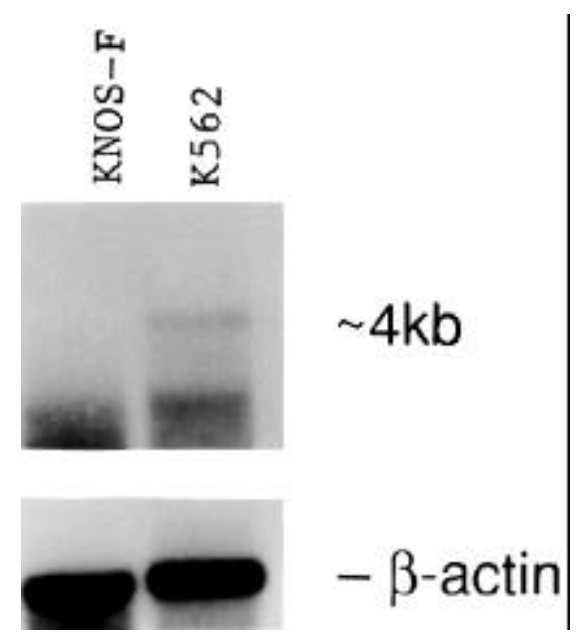

Figure 3. Northern blot prepared with mRNA isolated from K562 cells in the presence and absence of nitric oxide expression probed with radiolabelled D9U10. A 4 kilobase mRNA is identified in the K562 lane, but absent in the KNOS-F lane. Beta actin signals from the same Northern blot are shown as a control for loading.

\section{ACKNOWLEDGMENT}

We thank Helene F. Rosenberg for her support during this project, for technical advice, and for critical reading of this report.

\section{REFERENCES}

1. Innis, M. A., Gelfand, D.H., Sninsky, J.J., and White, T.J. PCR Protocols: A guide to methods and applications. Academic Press, New York (1990)

2. Bauer, D., Muller, H., Reich, J., Riedel, H., Ahrenkiel, V., Warthoe, P., and Strauss, M: Identification of differentially expressed mRNA species by an improved display technique (DDRT-PCR). Nucl Acids Res 21, 42724280 (1993)

3. Liang, P., and Pardee, A.B: Differential display of eukaryotic messenger RNA by means of the polymerase chain reaction. Science 257, 967-971 (1992)

4. Liang, P., Averboukh, L., and Pardee, A.B: Distribution and cloning of eukaryotic mRNAs by means of differential display: refinements and optimization. $\mathrm{Nucl}$ Acids Res 21, 3269-3275 (1993)

5. Domachowske, J. B., Rafferty, S.P., Singhania, N., Mardiney, M., and Malech, H.L: Nitric oxide alters the expression of - globin, H-ferritin, and transferrin receptor in human K562 cells at the posttranscriptional level. Blood 88, 2980-2988 (1996) 